\title{
Digital Transformation of Engineering Education
}

\author{
Empirical Insights from Virtual Worlds and Human-Robot-Collaboration \\ https://doi.org/10.3991/ijep.v6i4.6023 \\ Anja Richert, Mohammad Shehadeh, Freya Willicks, Sabina Jeschke \\ RWTH Aachen University, Aachen, Germany
}

\begin{abstract}
Cyber-physical systems and "Industry 4.0" will require future engineers to handle big data and complex, multidisciplinary problems as well as to collaborate with machines in "hybrid teams". As some work spaces will be virtualized or remotely controllable new communication skills and the knowledge of virtual worlds are necessary. Furthermore, working as a team with machines demands not only knowledge of mechanical engineering and machines but also an extended understanding of team working. To meet such challenges future engineers need to acquire new skills and qualification. This task does not only concern engineering students and trainees but also teachers for engineering. Questions about how to prepare for newly needed engineering competencies for the age of Industry 4.0, how to assess them and how to teach and train e.g. human-robotteams have to be tackled in future engineering education. The paper presents theoretical aspects and empirical results of a series of studies, which were conducted to investigate engineering education in virtual worlds as well as different aspects about team building in hybrid teams.
\end{abstract}

Index Terms-virtual worlds, human-robot-collaboration, engineering education, hybrid teams

\section{INTRODUCTION}

The fourth industrial revolution is characterized by new forms of artificial intelligence and by the omnipresent networking of "everything with everything" [1]. Thus, the training of virtual and joint problem solving within networks of avatars, virtual humans and machines is a huge field of research, in which empirical evidence is strongly needed.

In the classical production line, human and machine operate in serial or parallel work steps most of the time. Until now, robots are mainly located in security-restricted areas or in fully automated sections. The technological advances that constitute Industry 4.0 have the potential to change classical production lines to in-the-box production of multi-agent-systems operating on concepts of decentralized artificial intelligence. Humans and machines will be working as a team and physically next to each other. Parts of value chains are operated and maintained fully remote or on the basis of virtualized processes [1].

Working in the digitalized and networked production environment within virtual working spaces or in humanrobot-teams demands a different skillset as the classical industrial production setting. As in pure human teams, heterogeneous actors bring in their skills and strengths. Team structures evolve. Hybrid human-robot-teams work productive in a fully networked production environment and ensure the competitiveness of high-wage countries. Engineering Education has to face these challenge of delivering educational settings for an industrial revolution, which is just around the corner [2].

The paper derives the need for strengthening the education of competencies and qualifications, which help to tackle the challenges of Industry 4.0 - e.g. collaboration and problem solving in virtual worlds and human-robotteams (Section II.A) and delivers an overview about virtual worlds already used in education (Section II.B). The Sections III and IV give insights in the State-of the Art Research in Engineering Education and Human-RobotCollaboration. Section V shows the design of an empirical study currently carried out by the authors, which leads to insights into influences on task performance of humanrobot-teams. The paper ends with conclusions and an outlook on further research (Section VI).

\section{TRANSFORMATION OF ENGINEERING EDUCATION: FROM INDUSTRY 4.0 TO EDUCATION 4.0}

\section{A. Competencies and qualifications for future engineers}

Being a successful worker in Industry 4.0 presents specific questions to qualification needs of this new era of industrial labor. Not only the educational contents are under revision: The methods of skill development of the fourth industrial revolution have to meet the requirements of a new generation of employees and designers, who grew up with digital media and catch the "Working World 4.0" with altered competencies.

The innovation and development of cyber-physical systems will require computer scientists and network professionals to work with experts in various disciplines as well as in globalized contexts. This will revolutionize how universities educate engineers and scientists [3].

The solving of complex, multidisciplinary problems within changing teams poses a strong challenge. The innovative use of the available big data and turning analytical knowledge to competitive advantages are some of the key competencies needed to successfully orchestrate Industry 4.0 networks. A comprehensive amplification of the IT skills for the next engineering generation is required.

New communication and collaboration settings come up, as some of the workspaces will be fully virtualized or remotely controllable. Engineers are going to be part of hybrid human-robot-teams collaborating with natural user interfaces such as the head-mounted display OCULUS RIFT (see Fig. 1) or voice user interfaces to mention only two possible user interfaces.

Largely autonomous organized value chains lead to substantial new tasks for human labor. The interaction with intelligent machines provokes severe changes in competence profiles for the working force 4.0 and espe- 

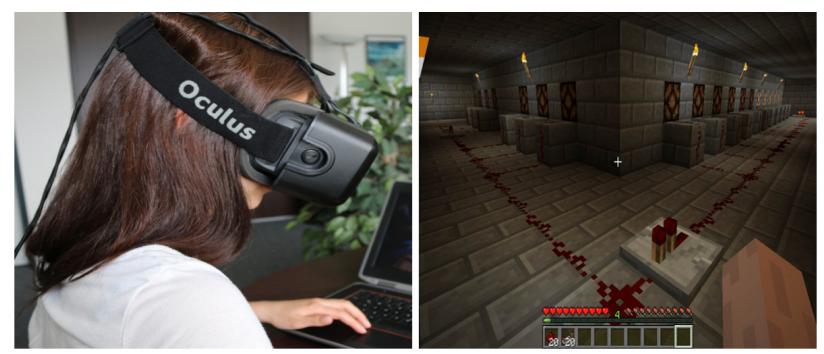

Figure 1. VLE used with Oculus Rift

cially for engineering staff. In the focus of needed competencies soft skills and interdisciplinary competencies gain importance. Through the interlinking of education and competence measurement, increasing demands on metacognitive and social skills arise. Beside of being an expert or specialist on certain subjects also the ability of global thinking, interdisciplinary knowledge and a holistic understanding of organizations becomes important.

Thus, the knowledge transfer within vocational training, higher education and advanced training is massively changing. Through the necessary co-working of disciplines, broad action fields arise and spread across the borders of standardized education. Companies become a more and more strong educational partner and a broker between academic and professional education systems. Lifelong Learning and the accreditation of qualification and competencies gained within non-formal learning scenarios such as virtual worlds become of utmost importance.

\section{B. Virtual Worlds in education}

Non-formal educational settings often derive based on existing virtual worlds from entertainment segments. Since a few years, the use of virtual environments such as Minecraft or Second Life has become a well-known leisure activity, mostly for the Generations $Y$ and $Z$ [2]. The challenge to utilize their fun and experience on virtual and game-based learning for educational purposes is also being addressed in some settings of school and higher education. A typical finding is that virtual learning environments (VLE) such as Second Life are more used in the domain of research and higher education and environments like Minecraft are more used within school education contexts as well as for engineering and IT hardware scenarios. [3].

Virtual reality solutions already contain a variety of specialized simulations for education and training purposes e.g. a vehicle simulator for the Federal German Armed Forces (see Fig. 2) [4]. The system named "KoCUA" (cooperative computer-aided training) is used since 2005 and comprises a virtual world for the training of bridge building and ferry services. Therefore, 3D models of amphibian vehicles, boats and floating bridges are available and have to be assembled to ferries and bridges. Features of this virtual world comprise variable configurations of training scenarios (e.g. flow, sight and wind conditions), monitoring view for trainers, data glove and real-time tracking of gestures.

For the training of fire and medical emergency situations a variety of virtual worlds exist. One of them is the transport and accident information and support system of the German Chemical Industry (see Fig. 3). Within five scenarios, firefighters can train their behavior on complex

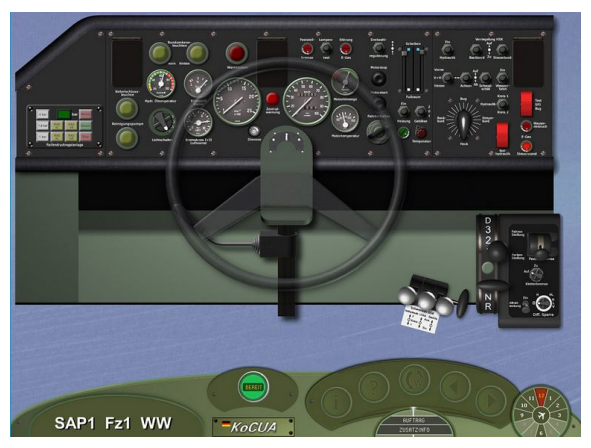

Figure 2. Virtual Training Space KoCUA [4]
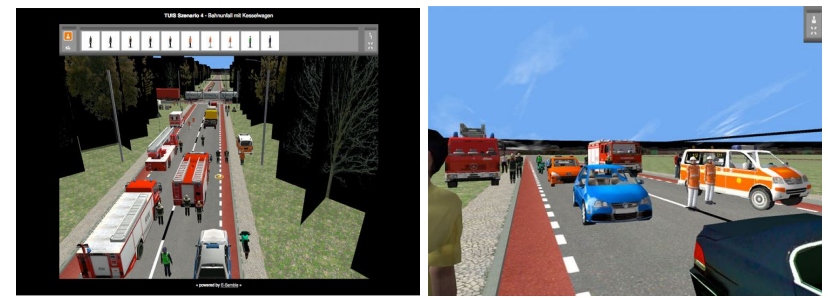

Figure 3. Virtual Training Space TUIS-VR [5]

transport accidents with dangerous goods on motorways, rails and country roads. Most of the firefighters have not been called very often to those accidents in their daily business. Within those Virtual Training Spaces they can train their behavior and to cope with those complex operations [4].

Empirical studies about the use of immersive Virtual Reality in learning sciences investigated the digital transformations of teachers, students and social context. It was shown that teachers with augmented social perception (i.e., receiving visual warnings alerting them to students not receiving enough teacher eye gaze) were able to spread their attention more equally among students than teachers without augmented perception. Other experiments showed that breaking the rules of spatial proximity that exist in physical space, students can learn more by being in the center of the teacher's field of view (compared to the periphery) and by being closer to the teacher (compared to farther away). Moreover, it was discovered that inserting virtual co-learners who were either model students or distracting students changed the learning abilities of experiment participants who conformed to the virtual co-learners. Results suggest that virtual environments will have a unique ability to alter the social dynamics of learning environments via transformed social interaction [4].

There is a broad variety of domains where creative problem solving is one of the key competences to be trained. Also the training of team competencies can also be set into virtual worlds. Most of the mentioned scenarios address settings, which you cannot train in real world scenarios to that extend - e.g. due to cost or danger reasons. For the implementation in higher education especially in engineering subjects, the question of the benefits for virtual training of every-day engineering tasks is raised to make the education of engineers in virtual labs and worlds a normal course and not an exception. Therefore, more insight in the learning and training process within virtual worlds is needed. The next section focusses on some useful insights from empirical studies. 


\section{EMPIRICAL STUDIES ON ENGINEERING EDUCATION} IN VIRTUAL WORLDS

In recent years, the usage of VLEs in engineering education has been significantly grown. The University of Ulster e.g. launched their Second Life Campus "Engineering Education Island" in 2006. The island consists of a welcome center, which includes general information on the project, and teleport links to the demonstrations and exhibits. "Each floor of the virtual laboratory contains a range of interactive engineering demonstrations and simulations. Other facilities on the Island include a virtual lecture theatre where students can attend classes and collaborative working facilities where students can work together remotely" [6]. A detailed description of the VLE can be found in Callaghan et al. [6], but there are no empirical data of the learning processes. Related work can also be found in Abulrub et al. [7].

To achieve a better understanding of virtual education a series of empirical studies was conducted by the authors. Explorative insights into training and problem solving processes within virtual worlds are given in the next sections. To install virtual education as a mean of classical higher education it has to be investigated if and to what extend informal learning-scenarios, like virtual worlds, are teachable and how teachers can transfer and implement their teaching ability within those environments. As there is a need for increased collaboration competencies and hands-on experience for engineers in Industry 4.0 the collaboration and problem solving behavior in anonymous teams within VLEs is also empirically explored.

In the "Study on Trainers' Perspective" professional trainers aged between 24 and 60 years (Mean = 40,7; Standard Deviation $=13,2$; n $($ sample size $)=10)$ participated [8]. To explore the trainers' perspective within the virtual world, every participating trainer had to fulfill two roles: first the trainee and then the trainer. The open-world game Minecraft was the setting for the VLE, a program that has been used successfully for learning and teaching purposes in the past and allows participants, amongst other things, the freedom to explore a virtual environment freely [8]. To link this research to the topic "Industry 4.0", a task with an engineering background was given to the participants who entered the virtual environment by wearing a head mounted display (OCULUS RIFT, DK 2; Figure 4).
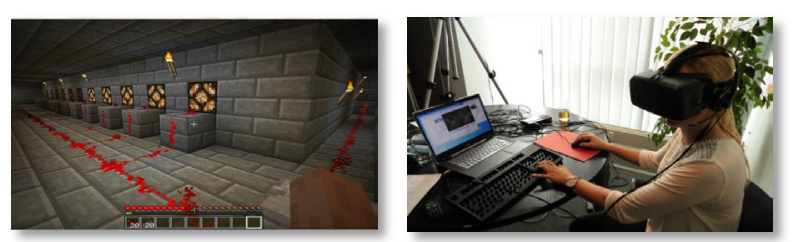

Figure 4. Left: Screenshot of the VLE, implemented in Minecraft, recorded by the video capture tool, Right: participant entering the VLE by wearing the OCULUS RIFT

During the experiment, the participants' spatial behavior within the VLE Minecraft was assessed by the use of a screen capture tool (see Fig. 5). After the experiment, a structured interview gave additional insights into the trainers' experiences in the VLE. For analysis purposes, three independent scientific analysts coded all the qualitative data of the videos. The data was then scored on a sixpoint scale (ranging from $1=$ low to $6=$ high). The scores of the three analyses were collapsed into one score for each variable and were then analyzed using SPSS.

The video data indicates that the age and online-gaming experience of participants are correlated (r) to the participants' spatial coordination within the VLE ( $\mathrm{r}$ age $=-.60$, $\mathrm{p}<.05 ; \mathrm{r}$ gaming $=.78, \mathrm{p}<.01)$. Older participants, and those who had no gaming experience showed initial difficulties with the hand-cursor coordination in the habituation phase; indicated by more questions, slower and less fluid movements and spatial problems. Participants who reported higher sensations of immersion, got used to the virtual world faster, as seen by their objective behavior within the VLE (e.g. number of gaze fixations ( $\mathrm{r}$ gaze $=.92, \mathrm{p}<.01)$, spatial coordination $(\mathrm{r}$ spatial $=.94, \mathrm{p}<.01)$, general task performance (efficiency) as a trainee and respectively as a trainer $(\mathrm{r}$ trainee $=.91, \mathrm{p}<.01)$; $(\mathrm{r}$ trainer $=.92, \mathrm{p}<.01)$ ).

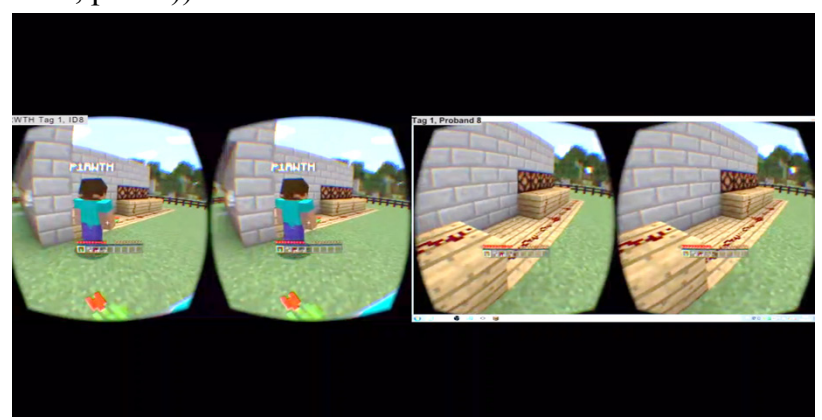

Figure 5. Screenshot of the VLE, implemented in Minecraft, recorded by the video capture tool, on the left side: trainers view, on the right side: trainees view

However, the question whether this is a consequence of participants' precondition or a result of mixed-reality devices could not be analyzed in this study and deserves further investigation. Participants' increased work experience in the field of training and coaching did partially affect their efficiency in the VLE, as seen by the participants' overt behavior and their communication skills. The trainer's evaluation of their previous experience within the VLE had a quite positive outcome. It indicates that barriers seem to be low regarding utilization of VLE for teaching and training purposes. The benefit of using virtual worlds for teaching purposes was rated as very helpful (Mean $=4.5$; Standard Deviation $=1$, rated on a five-point scale, ranging from $1=$ not helpful at all to $5=$ very helpful) and the same was said about the potential use of these worlds as a training method for a specific job (Mean $=5$; Standard Deviation $=0$ ) [8].

The participants reported feeling immersed in the virtual world and mentioned that they used and adapted specific learning methods whilst they were using the game, which lead to faster familiarization with the VLE. Regarding fields of further application, the trainers suggested this kind of simulation could be useful for applications within everyday schooling, labs in higher education, and in the training of techniques, which are too hazardous for the training in the physical reality [8].

Resource efficiency and flexibility, as well as the targeting of many senses at once were seen as added values of training with VLE. The possibility of changing or adapting single parameters for training were also mentioned as potential benefits of VLE. Potential difficulties with using virtual worlds for training purposes include aspects like the degree of acceptance of the technology or 
the blurred display one sees when looking into the distance and which can lead to simulator sickness. However, the benefits exceed the possible adverse effects and were reflected in the trainers' positive attitude towards this new training method.

A second study, also conducted by the authors, investigated in detail the aspects of collaborative problemsolving behavior in VLEs. The experimental design of this study consists of two groups. The experimental group had to fulfill the task at a laptop by wearing the OCULUS RIFT, a natural user interface that allows the user to control his field of view by natural head movement. Previous studies have shown that this has positive effects on immersion of the user [3]. The control group had to fulfill the same task at the laptop, controlling the field of view with a mouse. The participants got the same engineering task as the trainers before; the repair work of the electrical circuit. The two participants, who were located in different rooms, met each other for the first time in the VLE. The communication between them took place over headphones. Besides the influence of immersion and flow on collaboration, also the construct social presence was examined. Social Presence is an important determinant for participation and social interaction as well as a prerequisite for collaborative learning and knowledge management [4].

The collaboration behavior is examined by using a mixed-method-approach, in order to deal with the complexity of these research settings. Through the aid of screen captures and concurrent voice recording, a detailed analysis of communication, action and interaction is possible. A special tracking system records the spatial coordination for the collection of the participants' movement patterns, their level of physical activity as well as the mapping and comparison of motion path. In the run-up to the tutorial and the learning scenario, the participants were asked in a pre-test about physical limitation with reference to the used technique, experience with digital games und game controllers, personality traits, behavior roles in learning and work situations as well as acceptance for technology. Following the learning scenario, the participants were asked in a post-test about the acceptance of the previously used technique, questions about flow and immersion, social presence as well as evaluation of communication between team members. A structured interview supplemented the questionnaires in the fields of acceptance of technique, feelings of flow and immersion as well as team coherence qualitatively.

As the data analysis and interpretation is still not finished, the preliminary results already show that age and online-gaming experience were related to participants' spatial coordination within the VLE. It's very likely that students which have no or only a few experiences in online gaming need more guidance in VLE, which can be realized by adaptive designing of the scene as well as through adaptive teaching behavior.

Furthermore, the data show that participants who reported higher sensations of immersion, got used to the virtual world faster, as seen by their objective behavior (e.g. number of gaze fixations, fluency of movement). Additionally, the data reveal that participants who documented their course of action aloud performed more efficiently. Hence, they finished the sub-goals faster and transferred their knowledge into their problem-solving processes. In order to strengthen the learning process in VLE the tasks should foster the students to talk about their course of action with themselves as part of the selfreflection or each other as part of the team collaboration process.

Working together in virtual teams with changing team members is one scenario, which will take place in the fourth industrial revolution. Another aspect we need to prepare for and educate is the collaboration within teams of humans and robots, which will be an important skill for future engineers and worker within Industry 4.0.

\section{EXISTING STUDIES AND THEORETICAL ASPECTS ON HUMAN-ROBOT-COLLABORATION}

Human-robot-collaboration is considered as an asset in the foundation of Industry 4.0. The long-term goal is to work and cooperate with robots in dynamic, unpredictable and changing environments, responding to changes autonomously as a team and learning from the experience. Shared environment human-robot teaming scenarios are at the center of a growing field of robotics and AI research.

The domain of human-robot-collaboration is an interdisciplinary research field, constituted by topics like task planning, motion planning, intention recognition, user modeling, scene recognition, and human-robot communication, often established under the framework of the socalled "Social Robotics". These systems are expected to safely and efficiently perform complex actions, assisting humans and independently completing tasks, in a diverse range of scenarios with highly dynamic and uncertain environments [9].

There is a broad variety of recent studies, which aim at getting insights on the prerequisites of human-robotcollaboration. Most of them are based on empirical data gained during the development and testing of prototypic robots, build as demonstrators for research purposes. Inagaki et al. [10] researched the perception, recognition and intention inference that should be applied to the human-robot production systems to construct more comfortable industrial environments. The robot "Hadaly-2", researched and developed by Morita et al. [11], utilizes the surrounding environment for collaboration, and is capable of carrying an object to a target position by reacting to visual and audio instruction by the human while ensuring his safety.

Moreover, the understanding of special referencing of a robotic system is needed to realize natural human-robotcollaboration. To allow a robot to understand different reference systems the robot Ripley was created. The robot is capable of interpreting the environment from its perspective via sensor data or by integrating natural language data from its conversation partner. Using verbal communication, Ripley was able to understand the difference between spatial references such as distinguishing between its own left and the partner's left. The results illustrate the importance of situational awareness and a common frame of reference in spatial communication [12]. Rani et al. researched a robot that is able to capture the anxiety level of a human and adapt its behavior to this. To minimize biases and errors, the emotional state of the human is interpreted by the robot through physiological responses that are generally involuntary and are not dependent upon culture, gender or age [12].

$\mathrm{Fu}$ et al. [13] note that for humans and robots to work together as peers, the system must provide mechanisms for the humans and robots to communicate effectively. 
The Human-Robot Interaction Operating System introduced in their work enables a team of humans and robots to work together on tasks that are well defined and narrow in scope. The human agents are able to use spatial dialog to communicate and the autonomous agents use spatial reasoning to interpret 'left of' type elements from the spatial dialog. The ambiguities arising from such dialog are resolved through the use of modeling the situation in a simulator.

Robots are currently mostly seen as a support tool in achieving small tasks or as an automation alternative in carrying out complex tasks by themselves [10]. To convert the existing research results of social robotics to the joint problem solving of human-robot teams, some empirical studies and prototypic solutions are available as well. Research has shown that if robots shall be considered as a workmate and a team member, a common ground or platform for mutual and meaningful interactions should exist. A human-robot collaborative system should take advantage of varying levels of autonomy and multimodal communication allowing the robotic system to work independently and ask the human counterpart for assistance when a problem is encountered. Identifying and focusing the attention on the critical problems that arise greatly improves performance in collaborative work. Grounding, an essential ingredient of the collaboration model can be achieved through meaningful interaction and the exchange of dialogue [10].

There is still need for more empirical insights to educate engineers for Industry 4.0. It has to be investigated, what students require to accept robots as a learning partner and how to collaborate with them already within learning processes. Therefore, it is required to have a better understanding of student's mental models of interactive robots, of the attributes they expect robots to have, and of how their responses differ over contexts and tasks [12].

In the specific study described in the next section students and robots are working together on a learning task, while factors like trust, confidence and patience within the task fulfillment and their impact on teambuilding and relationship of human-robot-cooperation is observed.

\section{EMPIRICAL STUDY ON HUMAN-ROBOT-TEAMS}

The experimental study is carried out as part of the exercise of the lecture "Learning and working behavior in a digitalized society". The task is to let a human-robot-team change the $\mathrm{pH}$ value of a provided mixture from a random $\mathrm{pH}$ level to the $7.0 \mathrm{pH}$ neutral level. The robot used for the experiment is a LEGO ${ }^{\circledR}$ Mindstorms ${ }^{\circledR}$ EV3 that is programmed beforehand and is equipped with a $\mathrm{pH}$ sensor from Vernier. The size of the robot is considerably small as it has the dimensions $19 \mathrm{~cm} \times 21 \mathrm{~cm} \times 41 \mathrm{~cm}$ for length, width and height. A Student group of three and the robot interact with each other in order to complete the assignment. The processes of teamwork are investigated and values of trust, confidence and patience are quantified by programming the robot to execute some errors or some delays and therefore observing the reaction of the human and the change in behavior. The focus lies on the following research questions:

- Will the students trust themselves or the robot, blaming the mistake on their selves or on the robot?

- How will this affect the confidence of the students in taking the robot as a learning partner?
- What role will the delays play in affecting the humans' patience within the learning process?

- Will the robot be seen as a support or as a nuisance in this task?

With this experimental design, the processes of teamwork are investigated and aspects of teambuilding are quantified and therefore the reaction and cooperation behavior of the humans are observed. This stimulates and assesses techniques such as social learning [14]. It is also investigated how the student will interact with the robot depending on previous knowledge on human-robotinteractions, or just judging or relating through physical appearance in case prior knowledge does not exist [15; 16]. At the same time, it would be the learning experience for the participants to see how the communication and interaction with the robot takes place throughout the experiment. The study assesses the relationship between personal characteristics, robot characteristics and their moderating impact on task performance (see Fig. 6).

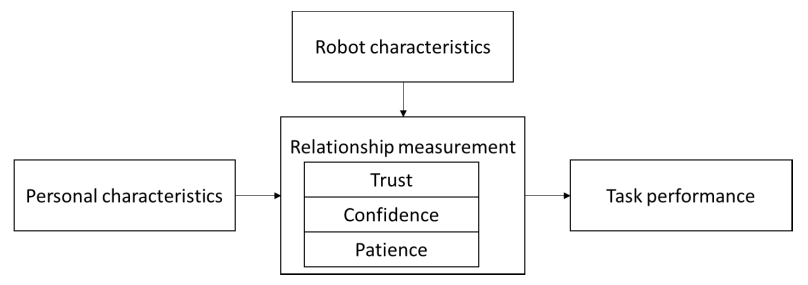

Figure 6. Independent, moderating and dependent variables of the experiment "pH measuring in hybrid teams"

The task scheme of the experiment can be described as follows: On a table, three beakers A, B and C are found. Beaker $\mathrm{A}$ is the main beaker of operations in the experiment. It contains a random solution of an unknown $\mathrm{pH}$ value of a substance. Initially beaker $A$ is half-full at 50 $\mathrm{ml}$ out of a total volume capacity of $100 \mathrm{ml}$. Beakers B and $\mathrm{C}$ contain solutions that may be added to beaker $\mathrm{A}$ for manipulating its $\mathrm{pH}$ value. Therefore, beaker $\mathrm{B}$ contains a highly concentrated solution with either a very high or very low $\mathrm{pH}$ value depending on what is in beaker $\mathrm{C}$. The experiment-part has a duration of 30 minutes and is videotaped. During the experiment the student are standing next to a Mindstorms robot on the same side of the table as illustrated in Figure 7. Beaker A is located between them on the table while beakers $\mathrm{B}$ and $\mathrm{C}$ are located on one end of the table closer to the test subject. The robot is able to provide the $\mathrm{pH}$ value of the solution in beaker $\mathrm{A}$.

There are two main scenarios with two student groups. The first scenario robot will behave perfectly without failure and in the second scenario the robot will be imperfect by carrying out different failures. Within the perfect scenario, the robot will communicate very clearly, promptly and accurately the $\mathrm{pH}$ values when asked. Within this imperfect scenario the robot will be very slow (30\% of the measurements take up to 40 seconds) and will make mistakes (in $25 \%$ of all measurements a wrong $\mathrm{pH}$ value is shown).

Since this experiment was only supposed to be a pretest for further studies about hybrid teams, the test sample can be considered as relatively small with only two test teams. It still delivers some useful aspects for further studies. The results base on the observations by the researchers during the experiment and the analysis of the 


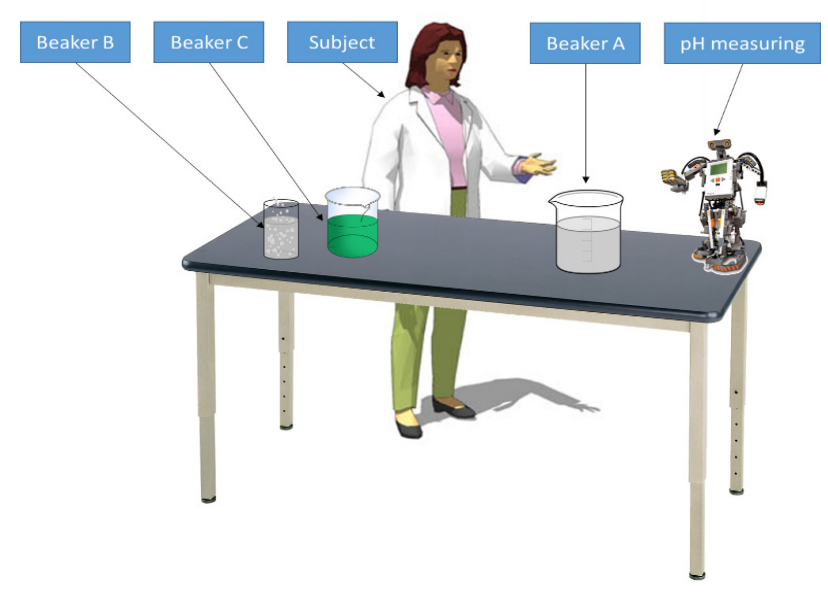

Figure 7. Experimental Setup of "pH measuring in hybrid teams"

videos. Overall, the task was finished faster by the group of the perfect scenario. The group with the imperfect robot did not complete the task of getting a neutral $\mathrm{pH}$ value in the allowed time at all. But in both subcategories the interaction with the robot in general was less than expected. For several participants the robot seemed just to be a tool than a team member. It was used like a normal $\mathrm{pH}$ sensor. This applies for the imperfect as well as for the perfect scenario. None of the participants talked to the robot like one would talk to one of the other team members. One participant (of a perfect scenario) talked about the robot during the experiment and stated "It would be helpful if he collects our data and gives some advice." As a conclusion only the ability of measuring the $\mathrm{pH}$-value and providing this information seems not to be enough for the students to consider the robot as a team member like the others.

The teams assigned tasks in the beginning (for example one team member was responsible for beaker $\mathrm{B}$, one for beaker $\mathrm{C}$ and one for measuring the fluid in beaker $\mathrm{A}$ with the help of the robot). This observation on labor division is well-established within research about teamwork and team building. But due to reasons of efficiency and productivity of the whole team the test subjects started to switch these roles during the experiment. This is also a well-known fact within this research field as several studies have revealed that a continuous dynamic role allocation is superior over a constant role strategy [17]. Regarding to the results of the described experiment this flexibility of role allocation is also a characteristic of humanrobot-teams. However, it is important to take account of the fact that the robot was not able to execute another task but could only measure the $\mathrm{pH}$ value.

In the imperfect scenario it took a quite long time until the team members realized that the robot was faulty. Only in the end of the experiment one participant stated the robot would behave falsely. Instead, most of them apparently tried to trust in the robot's abilities. As it seemed it was hard for the participants to accept that technology could be faulty or tricky. Nevertheless, the students did not clearly blame the mistakes on their own team members or on themselves. At some points they started to have self-doubts but these were not strongly pronounced. Since studies have shown that errors or mistakes within communication might alter the human's confidence [14], one can assume that this indicates matching results. For clear indication an extended experiment could be useful, for example where the whole task is constructed more difficult with more differentiated individual tasks. Therefore, a comparison of the results with findings of former studies in this special point would be more valid.

The results of this experiment still offer interesting first insights into teamwork processes in human-robot-teams. The most important aspect concerns the human's perception of the robot: When is he only a tool and at which point does he start being a team member? In this regard it is necessary to rethink the definition of a team member when a robot shall be considered as one: What do humans expect from him to call him a member of their team? What exactly makes the difference between a "tool" and "team member"? This concerns for example the robot's ability to overtake several different tasks as well as the communication between the robot and the human being.

\section{CONCLUSION AND OUTLOOK: CONSEQUENCIES FOR FUTURE ENGINEERING EDUCATION}

The experiments and studies on VLEs show that not only Industry but also education is already on its way to "4.0". The challenge lies in preparing students and workforce to cope with (fast) breakthroughs of technologies, new decentralized control paradigms, enhanced roles of artificial intelligence, large amounts of uncertainty and other ongoing changes. Soft skills competencies, which will gain more and more importance, will be the ability to solve problems by virtual teamwork and to be able to work in hybrid teams consisting of human and robots, working indispensable together.

The results and initial findings described show that those VLE bear a promising potential for successful implementation of virtual worlds within higher education. The teaching and training of qualifications within virtual scenarios and human-robot-teams strengthens literacies, which will be part of our daily professional life. Trainers have to be able to prepare VLEs as they prepare a PowerPoint-Presentation today. Furthermore, tomorrows teachers need technological competencies in order to interact with students in the VLE. This "digital literacy" comprises the design of VLE, experience in digital coaching and other joint problem solving in virtual worlds. It is going to be a mode of teaching to tutor and moderate groups of students in VLEs [3]. By the help of Learning Analytics, the gathered data of VLEs can be assessed and used to analyze and optimize learning processes.

The described results of the VR-studies allow insights into the transferability of problem-solving strategies from real-world scenarios into the behavior within virtual worlds. An open question is how this affects the transfer achievements and trade-offs from virtual to real world scenarios. The work on those above mentioned constraints is already in process. Researcher of the University of Southern California and the Facebook Company Oculus showed how the facial expression of a VR-Headset user can be tracked and drawn to a virtual character. With the help of a 3D camera, the systems track movements of the mouth. Movements of the upper facial region are tracked by stretch marks, which are integrated within the headset. Drawn together those datasets enable a 3D-picture of facial movements of the user, which can be used for the animation of virtual characters [20].

The way courses of study are currently accredited and competencies are assessed have to be rethought. The inclusion of informal and open learning scenarios in our 
educational schemes has to be investigated. The evaluation of knowledge and competencies gained in such learning environments presents another important research question. The understanding and empirical insight in these teaching and learning processes is one first step on the way to education 4.0. Fundamental design paradigms for online assessments, which e.g. take into account the need for a "warm-up" and habituation phase of virtual worlds need to be developed [21]. Moreover, the legal situation of e-assessments in virtual worlds have to be clarified.

By tackling the above mentioned challenges, education 4.0 offers new and promising perspectives on educating and training groups of people, who have been difficult to address with classical courses of e.g. vocational and further education. The realization of hands-on experience, the deployment and integration of competencies and experiences of the digitalized generations have the potential to lead to new successful educational settings for those who are not used to learning and difficult to reach, due to their age, educational background and qualification level. Industry 4.0 and Education 4.0 does not only threaten existing jobs but also offers huge chances to profit from this digital and it-driven revolution.

\section{REFERENCES}

[1] H. Kagermann, "Recommendations for implementing the strategic initiative INDUSTRIE 4.0“, in Acatech - National Academy of Science and Engineering, Frankfurt/Main, 2013.

[2] K. Schuster, K. Groß, R. Vossen, A. Richert, S. Jeschke, "Preparing for Industry 4.0 - Collaborative Virtual Learning Environments in Engineering Education", in Proceedings of the International Conference on E-Learning in the Workplace (ICELW 2015), New York, USA, 10-12 June 2015, 2015.

[3] A. Richert et al., "Learning 4.0. Virtual immersive engineering education", in Digital Universities: International Best Practices and Applications, 2/2015.

[4] Szenaris GmbH, Virtual-Reality-Teamtrainer, http://www.szenaris.com/366-0-Virtual-RealityTeamtraining.htm,1 [30.12.2015].

[5] XVR, Ausbilden, Trainieren und Üben auf einer Simulationsplattform http://tuisvr.xvrweb.com/willkommen/, [30.12.2015].

[6] MJ.Callaghan, K.McCusker, J.Lopez Losada, JG.Harkin and S.Wilson, "Teaching Engineering Education using Virtual Worlds and Virtual Learning Environments", in International Conference on Advances in Computing, Control, and Telecommunication Technologies, 2009. https://doi.org/10.1109/ACT.2009.80

[7] A. G. Abulrub, Attridge, A.N., "Virtual reality in engineering education: The future of creative learning", Global Engineering Education Conference (EDUCON), 2011 IEEE. https://doi.org/10.1109/educon.2011.5773223

[8] L. Plumanns et al., "Investigating Mixed-Reality Teaching and Learning Environments for Future Demands: The Trainers' Perspective," in Proceedings of the 18th International Academic Conferences, London. In Press. https://doi.org/10.1007/978-3-31942620-4_31

[9] J.N. Bailenson et al., "The Use of immersive vritual reality in the learning Science. Digital transformations of teachers, students and social contexts," in The Journal of Learning Sciences, vol. 17, pp. 102-141, 2008. https://doi.org/10.1080/10508400701793141

[10] Y. Inagaki et al., "Behavior-based intention inference for intelligent robots cooperating with human," in International Joint Conference of the Fourth IEEE International Conference on Fuzzy Systems and The Second International Fuzzy Engineering Symposium., Proceedings of 1995 IEEE Int. Vol. 3. IEEE, 1995.

[11] T. Morita et al., "Design and control of mobile manipulation system for human symbiotic humanoid: Hadaly-2," in Robotics and Automation, Proceedings of IEEE International Conference, vol. 2. IEEE, 1998. https://doi.org/10.1109/robot.1998.677287

[12] P. Rani et al., "Anxiety detecting robotic system - Towards implicit human-robot collaboration", Robotica, 22, (1), 2004, 85-95. https://doi.org/10.1017/S0263574703005319

[13] D. Fu et al., "Human systems modeling: specifying the behavior of computer-generated forces without programming," in Proceedings of the 35th conference on Winter simulation: driving innovation. Winter Simulation Conference, 2003.

[14] A.R. Wagner, "The role of trust and relationships in human-robot social interaction”, Diss., Georgia Institute of Technology, 2009.

[15] Shibata and K. Tanie, "Influence of a priori knowledge in subjective interpretation and evaluation by short-term interaction with mental commit robot," in Intelligent Robots and Systems, Proceedings of IEEE/RSJ International Conference, vol. 1. IEEE, 2000.

[16] J. Goetz and S. Kiesler, "Cooperation with a robotic assistant," in CHI'02 Extended Abstracts on Human Factors in Computing Systems, ACM, 2002.

[17] A. Mörtl et al., "The role of roles: Physical cooperation between humans and robots," in The International Journal of Robotics Research, vol. 31, no 13, pp. 1656-1674, 2012. https://doi.org/10.1177/0278364912455366

[18] J. Cook and T. Wall, "New work attitude measures of trust, organizational commitment and personal need non-fulfillment," in Journal of occupational psychology, vol. 53, Issue 1, 1980, pp. 39-52.

[19] C.S. Carver, "You want to measure coping but your protocol'too long: Consider the brief cope," in International journal of behavioral medicine, vol. 4, Issue 1, 1997, pp. 92-100. https://doi.org/10.1207/s15327558ijbm0401_6

[20] T. Simonite, "Oculus Rift Hack Transfers Your Facial Expressions onto Your Avatar”, MIT Technology Review, 2015. Online available: http://www.technologyreview.com/news/537566/oculus-rifthack-transfers-your-facial-expressions-onto-your-avatar/ [22.011.2015].

[21] K. Schuster, "Einfluss natürlicher Benutzerschnittstellen zur Steuerung des Sichtfeldes und der Fortbewegung auf Rezeptionsprozesse in virtuellen Lernumgebungen," Marburg: Tectum, 2014.

\section{AUTHORS}

Prof. Anja Richert is managing director of the Center for Learning and Knowledge Management (ZLW) in the Cybernetic-Cluster IMA/ZLW \& IfU at RWTH Aachen University, Germany (e-mail: anja.richert@ima-zlwifu.rwth-aachen.de).

Mohammad Shehadeh is researcher at the Center for Learning and Knowledge Management (ZLW) in the Cybernetic-Cluster IMA/ZLW \& IfU at RWTH Aachen University, Germany (e-mail: mohammad.shehadeh@ima-zlw-ifu.rwth-aachen.de).

Freya Willicks is researcher at the Center for Learning and Knowledge Management (ZLW) in the CyberneticCluster IMA/ZLW \& IfU at RWTH Aachen University, Germany (e-mail: freya.willicks@ima-zlw-ifu.rwthaachen.de).

Prof. Sabina Jeschke is director of the CyberneticCluster IMA/ZLW \& IfU and vice dean of the department of mechanical engineering at RWTH Aachen University, Germany (e-mail: sabina.jeschke@ima-zlw-ifu.rwthaachen.de).

This article is an extended and modified version of a paper presented at the EDUCON2015 conference held in Abu Dhabi, UAE, 10-13 April 2016. Submitted 09 July 2016. Published as resubmitted by the authors 13 August 2016. 